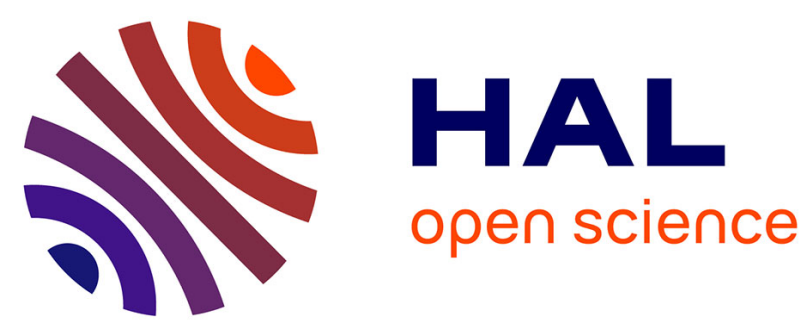

\title{
Performance evaluation of a TDMA-based multi-hop communication scheme for reliable delivery of warning messages in vehicular networks
}

\author{
Mohamed Hadded, Paul Mühlethaler, Anis Laouiti
}

\section{To cite this version:}

Mohamed Hadded, Paul Mühlethaler, Anis Laouiti. Performance evaluation of a TDMA-based multihop communication scheme for reliable delivery of warning messages in vehicular networks. IWCMC 2017 - 13th International Wireless Communications and Mobile Computing Conference, Jun 2017, Valencia Spain. pp.1029 - 1034, 10.1109/IWCMC.2017.7986427 . hal-01617924

\author{
HAL Id: hal-01617924 \\ https://hal.science/hal-01617924
}

Submitted on 17 Oct 2017

HAL is a multi-disciplinary open access archive for the deposit and dissemination of scientific research documents, whether they are published or not. The documents may come from teaching and research institutions in France or abroad, or from public or private research centers.
L'archive ouverte pluridisciplinaire HAL, est destinée au dépôt et à la diffusion de documents scientifiques de niveau recherche, publiés ou non, émanant des établissements d'enseignement et de recherche français ou étrangers, des laboratoires publics ou privés. 


\title{
Performance Evaluation of a TDMA-based Multi-hop Communication Scheme for Reliable Delivery of Warning Messages in Vehicular Networks
}

\author{
Mohamed Hadded*†, Paul Muhlethaler* and Anis Laouiti ${ }^{\dagger}$ \\ *INRIA, BP 105. 78153 Le Chesnay Cedex, Paris-Rocquencourt, France \\ †SAMOVAR, Télécom SudParis, CNRS, Université Paris-Saclay, 9 rue Charles Fourier 91011 EVRY, France \\ \{mohamed.elhadad, paul.muhlethaler\}@inria.fr, anis.laouiti@telecom-sudparis.eu
}

\begin{abstract}
Vehicular Ad hoc NETworks, known as VANETs, are deployed to reduce the risk of road accidents as well as to improve passenger comfort by allowing vehicles to exchange different kinds of data, both between the vehicles themselves and potentially between the vehicles and the infrastructure. One of the major issues in VANETs is the need to improve safety information delivery over long distances. Hence, VANETs require efficient and stable routing protocols that can allow the safety information to be disseminated in a timely manner. We recently proposed TRPM, a TDMA-aware routing protocol for multi-hop communication based on a cross layer approach between the Medium Access Control (MAC) and the routing layers, in which the intermediate vehicles are selected according to their geographic position and the position of their time-slot in the TDMA scheduling frame. The main purpose of this paper is to analyze the efficiency of the TRPM protocol. To do so an analytical model is presented in which expressions are derived to calculate two performance metrics: the delivery delay and packet loss rate. In order to validate the mathematical model and the protocol, a comparison between simulation and analytical results is presented using the network simulator ns-2 and the realistic road traffic simulator MOVE/SUMO.
\end{abstract}

\section{Introduction}

Vehicular Ad hoc NETworks, known as VANETs, are regarded as a promising communication technology that can meet various requirements of Intelligent Transportation System (ITS) applications which aim to help improve traffic safety and efficiency [2]. Through Vehicle-to-Vehicle (V2V) and Vehicle-to-Infrastructure (V2I) communications, each vehicle can exchange information to inform other vehicles about the current state of the traffic flow or the existence of a dangerous situation such as an accident. Road safety and traffic management applications require a reliable multi-hop communication scheme with minimal transmission delays and collisions, which increases the need for an efficient MAC and routing protocol. However, one of the major challenges of vehicular networks is to design an efficient multihop communication scheme which can cope with the hidden node problem, the high speed of the vehicles, the frequent changes in network topology, the lack of infrastructure, and QoS requirements of VANET safety applications [3].

Multi-hop communications in VANETs are mainly controlled by the decisions of the MAC layer and the network layer. However, when taken separately, these decisions may not be coherent, which can have an adverse effect on the delivery delay [5]. Moreover, most research has focused on solving the routing problems which are caused by the frequent topological changes in VANETs [6], [7] while much less attention has been paid to collision problems at the MAC layer. Therefore, it is possible that packet loss at the routing layer is mainly caused by data collision in the MAC layer, not by route failures. To tackle these problems, we recently proposed a cross layer protocol called TRPM [4], a TDMA-aware Routing Protocol for Multi-hop communication to ensure coherent decisions between the MAC and routing layers by selecting the next relay node according to the slot scheduling performed by the Distributed TDMA based MAC protocol, DTMAC [1]. To ensure the efficiency of relay selection, TRPM uses a weighted next-hop selection function in order to make coherent next hop decisions in terms of both the number of relay vehicles and end-to-end delay. The main goal of this work is to enable vehicles to send their event-driven safety messages over long distances in a timely manner and with a low collision rate.

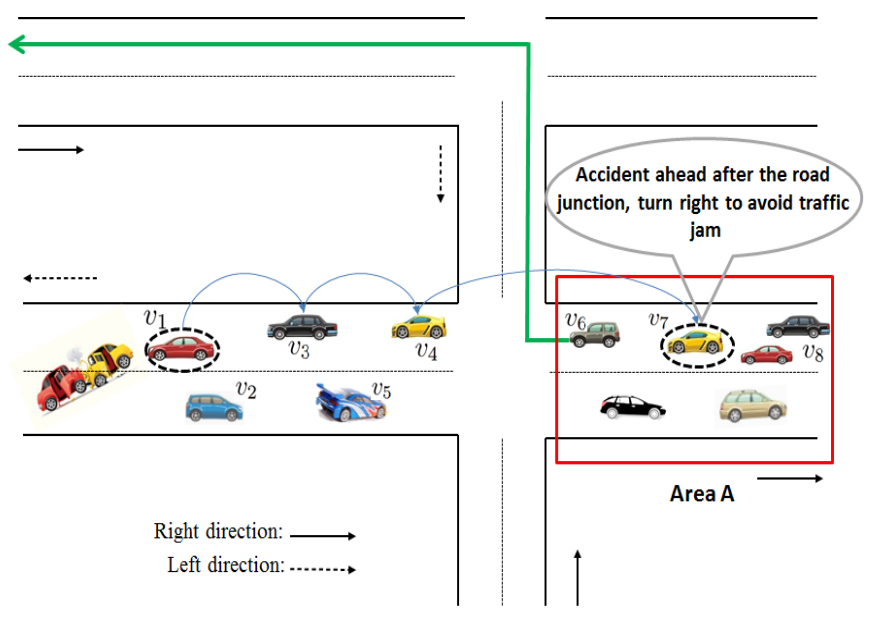

Figure 1. An accident detection by using the TRPM protocol.

Figure 1 shows an example of a safety application using the TRPM protocol. As illustrated in this figure, the vehicle $v_{1}$ has detected an accident in its area. In order to avoid the traffic jam, the vehicle $v_{1}$ will use the TRPM protocol to inform the vehicles that are moving in the area $A$ about 
this critical situation. The safety message will be routed in a unicast mode through relay nodes $v_{3}$ and $v_{4}$ to vehicle $v_{7}$ that will eventually broadcast the message received from vehicle $v_{1}$ in its own area. Upon reception of the message by a vehicle $v_{7}$, each vehicle in area $A$ will turn right in order to avoid the traffic jam. This paper presents an analytical model which evaluates two performance metrics, namely, the delivery delay and the packet loss rate. In addition, simulation results are given to validate the mathematical model and the protocol.

The rest of the paper is organized as follows. Section 2 describes the system model and briefly presents the DTMAC protocol [1] which is used as the MAC protocol in our proposal. Section 3 discusses details of our cross layer protocol, called TRPM [4]. The delay and packet loss analyses are given in Section 4 and 5, respectively. Section 6 presents the numerical results and the performance evaluation. Finally, conclusions and future work are reported in Section 7.

\section{DTMAC protocol}

DTMAC [1] is a distributed TDMA based MAC protocol for VANET which exploits the linear topology of VANETs. In DTMAC, as Figure 2 shows, the road is dissected into $N$ small fixed areas, denoted by $x_{i}, i=1, \ldots, N$, of length equal to $R$, where $R$ is the communication range of the vehicles. Area IDs can be easily derived using maps and GPS devices. The time slots in each TDMA frame are partitioned into three sets, $S_{0}, S_{1}$ and $S_{2}$, associated with vehicles in three contiguous areas: $x_{i}, x_{i+1}$ and $x_{i+2}$, respectively. Each frame consists of a constant number of time slots, denoted by $\tau$ and each time slot is of a fixed time duration, denoted by $s$. Each vehicle can detect the start time of each frame as well as the start time of a time slot. In the VANET studied here, all the vehicles are equipped with a GPS and thus the one-Pulse Per-Second (1PPS) signal that a GPS receiver gets from GPS satellites can be used for slot synchronization. In DTMAC, each vehicle moving in area $x_{i}$ knows only the slot scheduling information of its neighboring vehicles moving in area $x_{i+1}$ and $x_{i+2}$.

The TDMA scheduling mechanism of the DTMAC protocol uses the vehicle location and slot reuse concept to ensure that vehicles in adjacent areas have collision-free schedules in which the three subsets of time slots will be reused between neighboring areas in such a way that no vehicles in different adjacent areas can access the channel at the same time, and thus no interference can occur. To acquire this information, messages are exchanged between neighboring vehicles. Furthermore, specific information, called frame information (FI) is added to each transmitted packet to notify the neighboring vehicles of a time slot assignment. Each time slot is dynamically reserved by an active vehicle (a vehicle whose communication device is transmitting) for collision-free delivery of safety messages or other control messages. The FI consists of a set of ID Fields (IDFs) of size equal to the number of time slots per frame, $\tau$. Each IDF consists of three fields: VEC_ID, SLOT_STATUS and PKT_TYPE. The VEC_ID field contains the ID of the vehicle that is accessing the corresponding slot. The
SLOT_STATUS field contains the status of the slot which indicates whether the slot is idle, busy or in collision. Finally, the PKT_TYPE field indicates the type of packet transmitted by the vehicle, i.e. periodic information or event-driven safety messages. When an active vehicle $v$ moving within the area $x_{i}$ needs to acquire a time slot on the transmission channel, it starts listening to the channel during the set of time slots reserved for the area in which it is traveling, this set is $S_{j}(v)$, where $j=(i+2) \bmod 3$. At the end of the frame the vehicle $v$ can determine the set of its neighbors $N(v)$, the set of busy slots in $S_{j}(v)$, denoted by $B(v)$ and the set of available time slots denoted $F(v)$. Vehicle $v$ selects one slot in $F(v)$ at random.

\section{Multi-hop TDMA scheme for Inter-Vehicle Communications (IVC)}

Generally speaking, routing protocols proposed for VANETs are designed to find the best path for end-toend packet delivery, which can satisfy QoS requirements by considering the number of relay nodes and link lifetime. Although these protocols can achieve good performances in terms of the metrics studied, they are not simultaneously optimized to maximize the overall network performance. That is why we recently proposed TRPM [4], a TDMAaware Routing Protocol for real-time and Multi-hop communications which ensures coherent decisions between the $\mathrm{MAC}$ and routing layers by selecting the best next relay node according to the DTMAC scheduling scheme. The idea of TRPM is the following. Whenever a vehicle $i$ accessing the channel during the set $S_{k}$ wants to send/forward an eventdriven safety message, it constructs two sets of candidate forwarders based on its frame information (FI) as follows, where $T S(j)$ indicates the time slot reserved by vehicle $j$.

- $A_{i}=\left\{j \in N(i) \mid T S(j) \in S_{(k+1) \% 3}\right\} / /$ The set of vehicles that are moving in the adjacent right-hand area.

- $B_{i}=\left\{j \in N(i) \mid T S(j) \in S_{(k+2) \% 3}\right\} / /$ The set of vehicles that are moving in the adjacent left-hand area.

Each source vehicle uses the position of a packet's destination and the TDMA scheduling information to make packet forwarding decisions. When a source vehicle $i^{1}$ is moving behind the destination vehicle, it will select a next hop relay that belongs to set $B_{i}$; when the transmitter is moving in front of the destination vehicle, it will select a forwarder vehicle from those in set $A_{i}$. For each vehicle $i$ that will send or forward a message, we define the normalized weight function WHS (Weighted next-Hop Selection) which depends on the delay and the distance between each neighboring vehicle $j$. The $W H S_{i, j}$ value is calculated as follows:

$$
W H S_{i, j}=\alpha * \frac{\Delta t_{i, j}}{\tau}-(1-\alpha) * \frac{d_{i, j}}{R}
$$

where:

1. We suppose this vehicle moves from the right to the left 


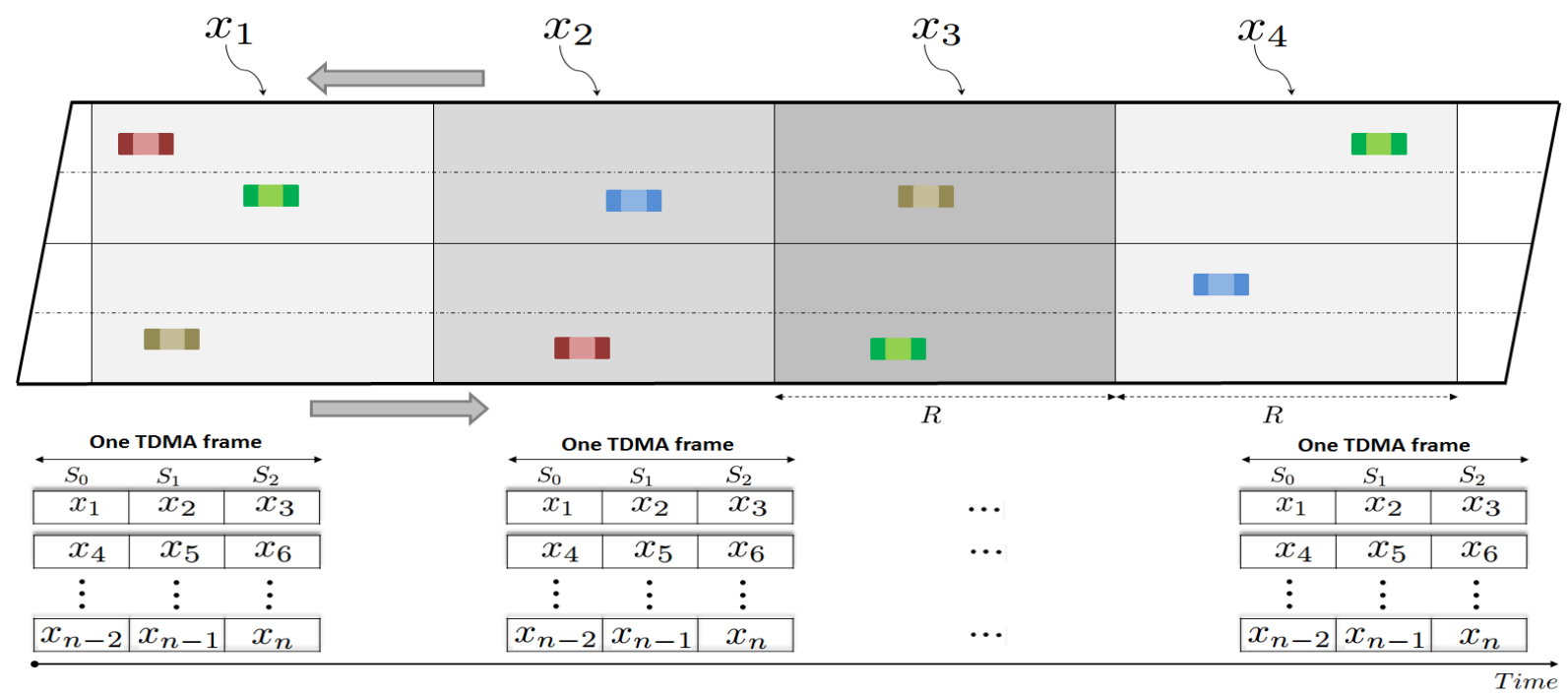

Figure 2. TDMA slots scheduling principle.

- $\tau$ is the length of the TDMA frame (in number of time slots).

- $\quad j$ is one of the neighbors of vehicle $i$, which represents the potential next hop that will relay the message received from vehicle $i$.

- $\Delta t_{i, j}$ is the time gap between the sending slot of vehicle $i$ and the sending slot of vehicle $j$.

- $d_{i, j}$ is the distance between the two vehicles $i$ and $j$, and $R$ is the communication range.

- $\quad \alpha$ is a weighted value in the interval $[0,1]$ that gives more weight to either distance or delay.

The forwarding vehicle for $i$ is the vehicle $j$ that is moving in the adjacent area in the direction of the destination and which has the lowest $W H S_{i, j}$ value. When a vehicle receives a message, it checks whether it is the destination of the packet, and if it is, it passes the packet to its upper layer. However, if the packet is intended for another vehicle, the receiver will check if the destination is moving in the same area, and if it is, the message will be transmitted immediately to its final destination. If the packet's destination is in another area, the receiver will calculate the next hop vehicle towards the destination. If a relay node is found, the message will be forwarded, if not, the message will be queued. Each forwarding vehicle includes its area ID in the relayed message. These steps are repeated by each relay vehicle until the packet is received by its final destination vehicle. To deliver a packet from a source to a destination, each vehicle $i$ that receives a message will use the weight function $W H S$ to select a forwarding vehicle in the next area from those listed in the set $A_{i}$ or $B_{i}$. By reading the area ID contained in the message it receives, the vehicle $i$ can determine the appropriate set of potential relays. It may happen that a node does not find any relay in the necessary adjacent area to forward its packet. In this case this node $i$ will look for an intermediate relay $j$ in its own area. We will also use the same general algorithm and select the relay that minimizes $W H S_{i, j}$. And $j$ will eventually relay the packet to a forwarder in the adjacent area.

\section{Delay analysis}

To obtain an analytical model with closed formulas for the delay and the packet loss in TRPM we must simplify the protocol and assume $\alpha=0$. In other words, in the forwarding process, the progression towards the final destination is the only criteria to select the next hop.

We assume that the nodes (vehicles) are distributed according to a 1D Poisson Point Process with rate $\lambda$. The road is divided into sections of $R$ meters, see Figures 3 and 4 . The slots are divided into frames of duration $\tau$. When a vehicle is granted access, a slot is acquired and repeated with a period $\tau$. The slot frame is divided into three sub frames of duration $\tau / 3$. The slots in a sub-frame are used by vehicles on the same section of the road.

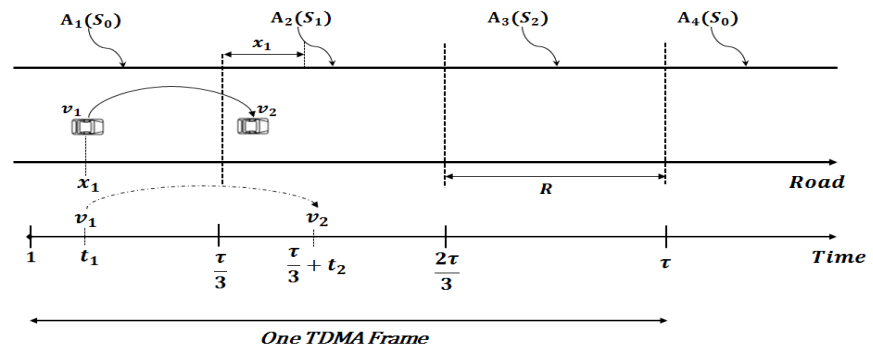

Figure 3. Next relay selection (first case).

The aim of this section is to evaluate the delay $D$ between the relay of the packet by a vehicle in one section and the relay of this packet towards the destination in the adjacent section and to compute the average of $D$ denoted $E(D)$.

We assume that our random vehicle $v_{1}$ is in section $A_{1}$ at the position $x_{1} \in[0, R]$ on the road and has acquired a slot $t_{1} \in\left[0, \frac{\tau}{3}\right]$. 
Conditioned by the fact that there is a relay vehicle in $A_{2}$ within transmission range of $v_{1}$. The mean number length of time before the next retransmission is :

$$
\frac{\tau}{3}-t_{1}+t_{2} .
$$

where $t_{1}$ is uniformly distributed in $\left[0, \frac{\tau}{3}\right]$. The probability of such an event is :

$$
1-e^{-\lambda x_{1}} \text {. }
$$

We now suppose that there is no relay vehicle in $A_{2}$ within transmission range of $v_{1}$. This occurs with probability $e^{-\lambda x_{1}}$. Then the protocol relies on an intermediate relay i.e. the furthest one in $A_{1}$. Such a vehicle exists with probability $1-e^{-\lambda\left(R-x_{1}\right)}$. Then we need to distinguish two cases:

- The intermediate relay vehicle in $A_{1}$ has a slot in $\left[t_{1}, \frac{\tau}{3}\right]$ in which case the delay to the next retransmission in $A_{2}$ is as in the previous case $\frac{\tau}{3}-t_{1}+t_{3}$ we only have the presence of an intermediate relay in $A_{1}{ }^{2}$. The probability of such an event is $1-\frac{3 t}{\tau}$.

- The intermediate relay vehicle in $A_{1}$ has a slot in $[0, t]$ in which case ${ }^{3}$ the delay to the next retransmission in $A_{2}$ is $\frac{\tau}{3}-t+\tau+t_{1}=\frac{4 \tau}{3}-t+t_{1}$. The probability of such an event is $\frac{3 t}{\tau}$.
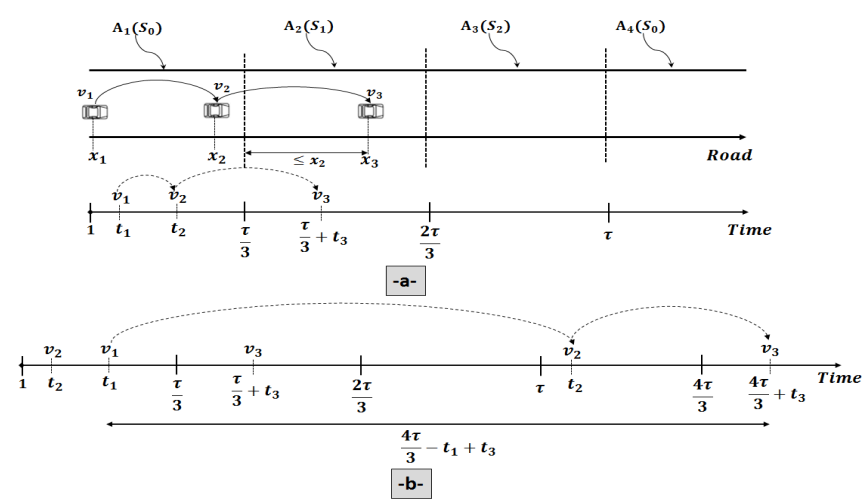

Figure 4. Next relay selection (second case).

Thus the delay $D$ contains three terms corresponding to the three cases described above.

$$
\begin{aligned}
D & =\left(1-e^{-\lambda x_{1}}\right)\left(\frac{\tau}{3}-t_{1}+t_{2}\right) \\
& +e^{-\lambda x_{1}}\left(1-e^{-\lambda\left(R-x_{1}\right)}\right)\left(1-\frac{3 t_{1}}{\tau}\right)\left(\frac{\tau}{3}-t_{1}+t_{3}\right) \\
& +e^{-\lambda x_{1}}\left(1-e^{-\lambda\left(R-x_{1}\right)}\right) \frac{3 t_{1}}{\tau}\left(\frac{4 \tau}{3}-t_{1}+t_{3}\right)
\end{aligned}
$$

In $D$ we have three random variables $t_{1}, t_{2}, t_{3}$ and $x_{1}$. According to the specification of the protocol, these random variables are independent. $t_{1}, t_{2}, t_{3}$ are uniformly distributed in $\left[0, \frac{\tau}{3}\right]$ whereas $x_{1}$ is uniformly distributed in $[0, R]$.

2. $t_{2}$ is only changed in $t_{3}$ since the slot of the relay in the next section is now denoted by $t_{3}$.

3. $v_{2}$ must wait a complete slot frame of length $\tau$ before serving as an intermediate relay toward $v_{3}$, see Figure 4 .
We obtain the following formulas:

$$
\begin{gathered}
E_{t_{1}, t_{2}, x_{1}}\left[\left(1-e^{-\lambda x_{1}}\right)\left(\frac{T}{3}-t_{1}+t_{2}\right)\right]=\left(1+\frac{e^{-\lambda R}-1}{\lambda R}\right) \frac{\tau}{3} \\
E_{t_{1}, t_{3}, x_{1}}\left[e^{-\lambda x_{1}}\left(1-e^{-\lambda\left(R-x_{1}\right)}\right)\left(1-\frac{3 t_{1}}{\tau}\right)\left(\frac{\tau}{3}-t_{1}+t_{3}\right)\right]= \\
=\frac{1-\lambda R e^{-\lambda R}-e^{-\lambda R}}{\lambda R} E_{t_{1}}\left[\left(1-\frac{3 t_{1}}{\tau}\right)\left(\frac{\tau}{2}-t_{1}\right)\right] \\
=\frac{\left(1-\lambda R e^{-\lambda R}-e^{-\lambda R}\right)}{\lambda R} \frac{7 \tau}{36}
\end{gathered}
$$

$$
\begin{aligned}
& E_{t_{1}, t_{3}, x_{1}}\left[e^{-\lambda x_{1}}\left(1-e^{-\lambda\left(R-x_{1}\right)}\right)\left(\frac{3 t_{1}}{\tau}\right)\left(\frac{4 \tau}{3}-t_{1}+t_{3}\right)\right]= \\
& =\frac{1-\lambda R e^{-\lambda R}-e^{-\lambda R}}{\lambda R} E_{t_{1}}\left[\frac{3 t_{1}}{\tau}\left(\frac{3 \tau}{2}-t_{1}\right)\right] \\
& =\frac{\left(1-\lambda R e^{-\lambda R}-e^{-\lambda R}\right)}{\lambda R} \frac{23 \tau}{36}
\end{aligned}
$$

Thus we have:

$$
\begin{aligned}
E(D) & =\left(1+\frac{e^{-\lambda R}-1}{\lambda R}\right) \frac{\tau}{3} \\
& +\frac{\left(1-\lambda R e^{-\lambda R}-e^{-\lambda R}\right)}{\lambda R} \frac{10 \tau}{12}
\end{aligned}
$$

\section{Packet loss analysis}

Here, in contrast to our previous description in Section 3 and to simplify the analysis we assume that a packet is lost as soon the protocol can not find any relay (or intermediate relay) toward the destination.

For each hop from one section of the road to the following, a packet is lost if first there is no relay in the next section within a transmission range of radius $R$ and if:

- there is no intermediate relay towards the destination in the same section (this is first event).

- or there is an intermediate relay in the same section but this intermediate relay has no relay towards the destination in the next section within its transmission range (this is the second event).

The probability of the first event is:

$$
p_{1}=e^{-\lambda x_{1}} e^{-\lambda\left(R-x_{1}\right)}=e^{-\lambda R}
$$

The density distribution of the distance $y$ between the intermediate relay and the next road section is $\lambda e^{-\lambda y}$. Thus the probability of the second event is:

$$
\begin{aligned}
p_{2} & =e^{-\lambda x_{1}}\left(1-e^{-\lambda\left(R-x_{1}\right)}\right) \int_{R-x_{1}}^{R} e^{-\lambda(R-y)} e^{-\lambda y} \lambda d y \\
& =e^{-\lambda x_{1}}\left(1-e^{-\lambda\left(R-x_{1}\right)}\right) \lambda x_{1} e^{-\lambda R}
\end{aligned}
$$


If we average $p_{2}$ on $x_{1}$ uniformly distributed in $[0, R]$, we obtain:

$$
\begin{aligned}
\overline{p_{2}} & =E_{x_{1}}\left[e^{-\lambda x_{1}}\left(1-e^{-\lambda\left(R-x_{1}\right)}\right) \lambda x_{1} e^{-\lambda R}\right] \\
& =\frac{\left(2 e^{\lambda R}-\lambda^{2} R^{2}-2 \lambda R-2\right) e^{-2 \lambda R}}{2 \lambda R}
\end{aligned}
$$

Thus from one section to the other, the probability of losing a packet because relaying is not possible according to the relaying protocol is:

$$
p=e^{-\lambda R}+\frac{\left(2 e^{\lambda R}-\lambda^{2} R^{2}-2 \lambda R-2\right) e^{-2 \lambda R}}{2 \lambda R}
$$

\section{Simulation results}

In this section, we provide the simulation and analytical results to prove the correctness of our analysis.

\subsection{Simulation scenarios and parameters}

We use MOVE [8] to generate vehicular traffic scenarios and SUMO [9] to perform real vehicular mobility simulations (see Figure 5).

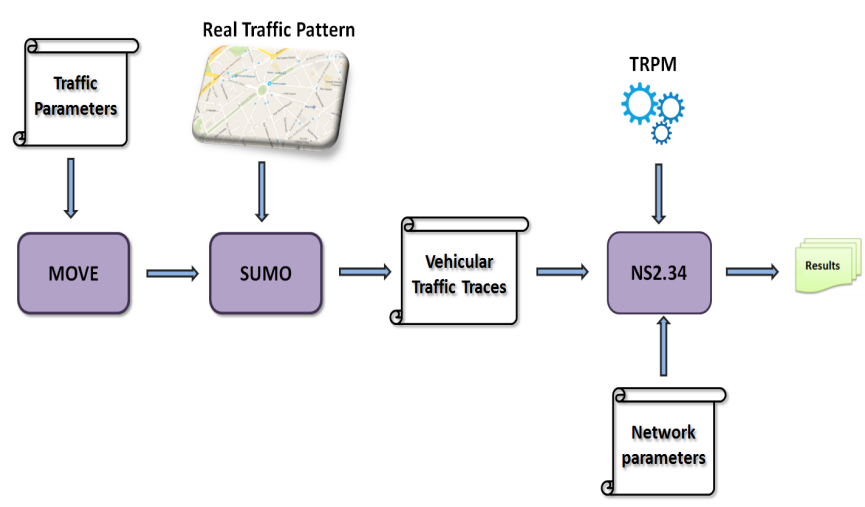

Figure 5. Simulation framework.

We generated a realistic VANET environment by selecting a real highway area from a digital map which took into account lane directions. Figure 6 shows a metropolitan area from the Map of San Jose (California) of size $3000 \mathrm{~m} \times 100 \mathrm{~m}$ exported from OpenStreetMap (OSM) and edited using Java OpenStreetMap Editor (JOSM). Then, we defined a vehicle flow which described a swarm of vehicles in each direction. The parameters of each vehicle flow consisted of the maximum number of vehicles, the starting road and destination of the flow and the time to start and end the flow. We assigned a random speed to each vehicle between $120 \mathrm{~km} / \mathrm{h}$ and $150 \mathrm{~km} / \mathrm{h}$. Then, the traffic traces generated by MOVE were used in the $n s 2.34$ simulator. The simulation parameters used in our experiments are summarized in Table 1.

Each simulation run lasts for 120 seconds. After the first 2 seconds of simulation, the source vehicle starts to transmit an emergency message 50 bytes in size. The message is transmitted to only one destination vehicle through multiple

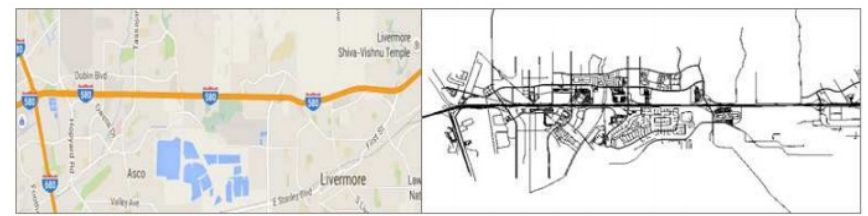

Figure 6. San Jose (California) urban area (left) exported to a VANET network topology by using MOVE/SUMO (right).

Table 1. Simulation parameters

\begin{tabular}{cc}
\hline Parameter & Value \\
\hline Highway length & $2 \mathrm{~km}$ \\
Lanes/direction & 2 \\
Vehicle speed & $120 \mathrm{~km} / \mathrm{h}$ \\
Speed standard deviation $(\sigma)$ & $30 \mathrm{~km} / \mathrm{h}$ \\
Transmission range & $310 \mathrm{~m}$ \\
Slots/frame & 100 \\
$n_{0}$ & $\tau / 3$ \\
$n_{1}$ & $\tau / 3$ \\
$n_{2}$ & $\tau / 3$ \\
Slot duration & $0.001 \mathrm{~s}$ \\
Simulation time & $120 \mathrm{~s}$ \\
\hline
\end{tabular}

relay nodes and is repeated periodically after one second. As shown in Figure 6, we consider a linear VANET topology $2 \mathrm{~km}$ long with a transmission range $R$ equal to $310 \mathrm{~m}$. The highway scenario consisted of 7 areas numbered from 1 to 7.

\subsection{Simulation results}

We have used a parameter, called area occupancy (AO) [1], equal to $N_{v} \times \frac{R}{L_{h}} \times \frac{1}{T_{s}}$ in a highway scenario, where $N_{v}$ is the total number of active vehicles, $R$ is the communication range, $L_{h}$ is the length of the highway and $T_{s}$ is the number of slots reserved for each area.

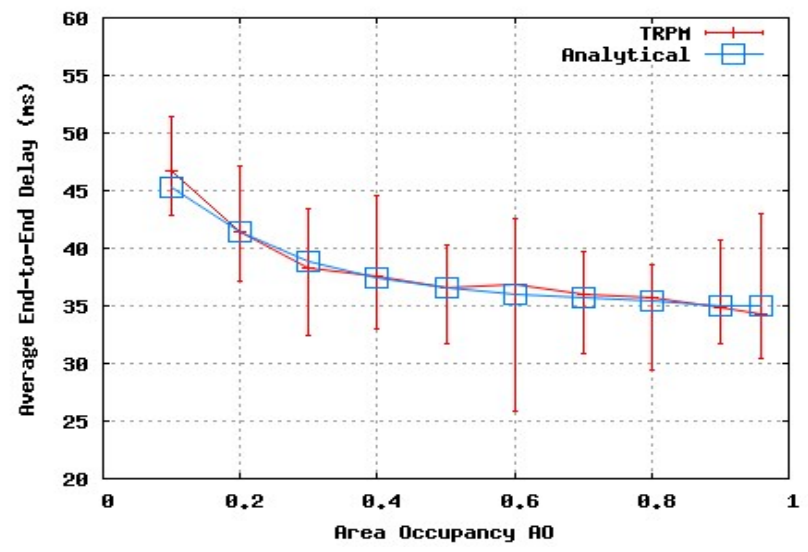

Figure 7. Average End-to-End Delay (ms) of a two-hop network vs vehicle density.

We simulated several scenarios by varying the area occupancy between 0.1 and 0.96 , which corresponds to traffic 
flow conditions varying from 22 to 204 vehicles in the whole network. Figure 7 shows the average end-to-end delay for a two-hop network when varying the Area Occupancy (AO). It is clear from this figure that the average end-toend delay decreases as the vehicle density increases. This is due to the fact that in high density networks there are more potential relays in the next area having small delays and greater distance progression towards the final destination. This figure shows the simulation and analytical results of the end-to-end delay of the TRPM protocol. We observe that the simulation results closely match the theoretical values for all $\mathrm{AO}$ values, which validates the simulation and analytical results.

The packet loss rate as a function of $\mathrm{AO}$ is plotted in Figure 8. As shown in the figure, the simulation results match almost perfectly with analytical results for the different densities considered. We can note that both the analytical model and the simulation show a packet loss rate equal to $0 \%$ when $A O>0.3$. These results can be explained by the fact that TRPM is a contention-free routing protocol which uses TDMA at the MAC layer thereby providing a collisionfree schedule.

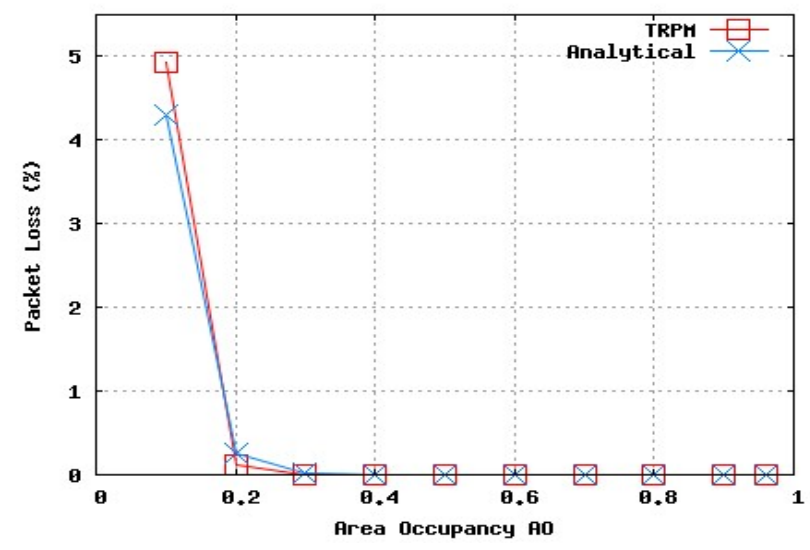

Figure 8 . Packet loss rate $(\%)$ of a two-hop network vs vehicle density.

To verify our analysis in a multi-hop vehicular network, we have considered multi-hop paths (See Figure 9). This figure shows the average end-to-end delay when varying the distance between the source and the destination between $550 \mathrm{~m}$ and $1750 \mathrm{~m}$. It is clear that the average delay increases as the distance increases. This figure compares the theoretical values of the average end-to-end delay with those obtained by simulation. We can note that the theoretical values are close to the simulated values for all the source-todestination distances shown. Hence, the simulation results and analytical calculations verify the accuracy of our analysis.

\section{Conclusion}

End-to-end delay and packet loss are the two major challenges in vehicular ad hoc networks. Since vehicular networks are deployed to improve road safety and efficiency

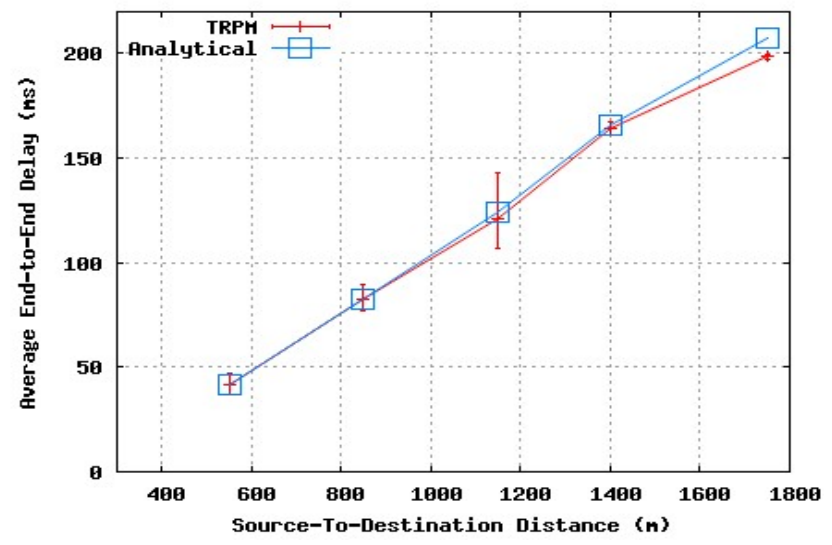

Figure 9. Average End-to-End Delay (ms) of a multi-hop network vs Source-to-Destination distance for $A O=0.2$.

by reducing the number of potential accidents, it is essential to ensure that all the vehicles can send their safety messages with a short end-to-end delay and a low packet loss ratio. In this paper, we presented an analytical model to evaluate the packet loss rate and the end-to-end delay for safety messages transmitted in vehicular networks over long distances when TRPM is used as a routing protocol. Comparisons of realistic simulation results, carried out using ns-2 and MOVE/SUMO, and analytical results show that the analytical model proposed provides close approximations for the end-to-end delay and packet loss rate for the different scenarios considered in this paper.

\section{References}

[1] M. Hadded, A. Laouiti, P. Muhlethaler, and L. A. Saidane, An infrastructure-free slot assignment algorithm for reliable broadcast of periodic messages in vehicular ad hoc networks, in Vehicular Technology Conference VTC-Fall, Montreal, Canada, Sep. 2016.

[2] P. Papadimitratos, A. d. L. Fortelle, K. Evenssen, R. Brignolo and S. Cosenza, Vehicular Communication Systems: Enabling Technologies, Applications, and Future Outlook on Intelligent Transportation, IEEE Communication Magazine , vol. 47, no. 11, pp. 84-95, 2009.

[3] M. Hadded, P. Muhlethaler, A. Laouiti, R. Zagrouba, and L. A. Saidane, TDMA-based MAC protocols for vehicular ad hoc networks a survey, qualitative analysis and open research issues, IEEE Communications Surveys Tutorials, vol. 17, no. 4, pp. 2461-2492, Jun. 2015.

[4] M. Hadded, A. Laouiti, P. Muhlethaler, and L. A. Saidane, TDMA aware Routing Protocol for Multi-hop Communications in Vehicular Ad Hoc Networks, IEEE Wireless Communications and Networking Conference IEEE WCNC, San Francisco, USA, March 2017.

[5] L. Louail, V. Felea, Latency optimization through routing-aware time scheduling protocols for wireless sensor networks Computers and Electrical Engineering, vol. 56, pp. 418-440, 2016.

[6] I. Leontiadis, C. Mascolo, GeOpps: geographical opportunistic routing for vehicular networks, WoWMoM 2007, Helsinki, Finland, 2007.

[7] J. Bernsen, D. Manivannan, RIVER: A reliable inter-vehicular routing protocol for vehicular ad hoc networks, Computer Networks. vol. 52, no. 17, pp. 3795-3807, 2012.

[8] F. Karnadi, Z. Mo, and K. chan Lan, Rapid generation of realistic mobility models for VANET, in IEEE WCNC, Hong Kong, China, pp. 2506-2511, Mar. 2007.

[9] SUMO Simulator: http://sumo.sourceforge.net. 